\title{
Identification of Floral Fragrances and Analysis of Fragrance Patterns in Herbaceous Peony Cultivars
}

\author{
Chaowei Song ${ }^{1}$ \\ Fuyang Vocational and Technical College, Fuyang 236031, People's Republic of China; and College \\ of Landscape Architecture, Beijing Forestry University, Beijing 100083, People's Republic of China \\ Qi Wang ${ }^{1}$ \\ College of Landscape Architecture, Beijing Forestry University, Beijing 100083, People's Republic \\ of China
}

Jaime A. Teixeira da Silva

P.O. Box 7, Miki-cho Post Office, Ikenobe 3011-2, Kagawa-ken 761-0799, Japan

\begin{abstract}
Xiaonan $\mathbf{Y u}^{2}$
College of Landscape Architecture, Beijing Forestry University, Beijing 100083, People's Republic of China; and Beijing Key Laboratory of Ornamental Plants Germplasm Innovation and Molecular

Breeding, National Engineering Research Center for Floriculture, Beijing 100083, People's Republic of China
\end{abstract}

\begin{abstract}
Additional Index words. Paeonia, aromatic compounds, DHS, GC/MS, clustering
Abstract. Thirty herbaceous peony (section Paeonia of the genus Paeonia) cultivars were divided into four groups (no fragrance, light fragrance, medium fragrance, or intense fragrance) based on their sensory evaluation scores. Using dynamic headspace sampling (DHS) and automatic thermal desorption-gas chromatography/mass spectrometry (ATD-GC/MS), 130 volatile organic components were detected in these 30 cultivars and a total of 72 compounds were identified as scent components. The main compounds were phenylethyl alcohol, $\beta$-caryophyllene, linalool, $(R)$ citronellol, and nerol. Selecting $\alpha$-pinene as the standard, the volatile components of these cultivars were quantitatively analyzed. By combining the sensory evaluation scores and the results of quantitative analysis, we found that 'Going Bananas', 'Cream Delight', 'Zhu Sha Pan', 'Qiao Ling', 'Duchess de Nemours', and 'Yang Fei Chu Yu' displayed an intense fragrance and, thus, had relatively high commercial value for the flower fragrance industry. 'Red Magic', 'Joker', 'Fairy Princess', 'Lovely Rose', 'Carina', and 'Etched Salmon' were excluded from the hierarchical cluster of aromatic compounds and the analysis of fragrance patterns because of the low amount of fragrance they released and poor sensory evaluation results. Based on a cluster analysis, assessment of the major aromatic compounds, and the results of sensory evaluation, the remaining 24 cultivars were divided into five fragrance patterns for the first time: woody scent [cluster I (major fragrance $\beta$-caryophyllene)], fruity scent [cluster II (phenylethyl alcohol)], lily scent [cluster III (linalool)], rose scent \{cluster IV [(R)-citronellol]\}, and an orange blossom scent [cluster V (nerol)].
\end{abstract}

Herbaceous peony, a perennial herb with a cultivation history exceeding 3000 years, is a famous traditional Chinese flower ( $\mathrm{Li}, 1999)$. It displays graceful petals and abundant colors, and many cultivars have elegant fragrance, an important characteristic of ornamental plants. The breeding of aromatic ornamental plants has involved research on aromatic composition and genetic mechanisms, including in Cyclamen persicum (Ishizaka et al., 2002), Petunia hybrida (Nakamura et al., 2006), Camellia japonica (Fan et al., 2006), Rosa rugosa (Feng et al., 2008), Osmanthus fragrans (Cao et al., 2009), Prunus mume

\footnotetext{
Received for publication 16 Apr. 2018. Accepted for publication 7 June 2018. This work was financially supported by the National Natural Science Foundation of China (31400591), Key Project of Natural Science Research of Anhui Provincial Department of Education (KJ2015A433), Local High-level University Project Construction Fund of Anhui Province (2015gx014), and Anhui Provincial Quality Engineering Project "Landscape Engineering Technology Teaching Team" (2015jxtd062). We would like to thank Liangsheng Wang (Beijing Botanical Garden, Institute of Botany, Chinese Academy of Sciences) for providing selfless guidance of this study.

${ }^{1}$ These authors contributed equally to this work.

${ }^{2}$ Corresponding author. E-mail: yuxiaonan626@126.com.
}

(Zhao et al., 2010), Chimonanthus praecox (Xiong et al., 2012), Chrysanthemum morifolium (Xu et al., 2012), Lilium species (Zhang et al., 2013), Hosta species (Liu et al., 2015), Hemerocallis species (Jiao et al., 2016), and Prosthechea cochleata (Ray et al., 2018). However, studies of herbaceous peony have focused primarily on species classification and phylogenetic analysis (Cheng et al., 2011; Guo and Wang, 2005, 2007; Hu et al., 2011; Yu et al., 2006), postharvest physiology of cut flowers (Kamenetsky et al., 2003; Li et al., 2007b; Shi et al., 2008; Wang et al., 2005, 2006; Wei et al., 2007), and the mechanism of flower color formation (Jia et al., 2008a; Zhao et al., 2012a), but surprisingly little attention has been paid to floral fragrance. A comparative analysis of the composition and content of the floral scent of 21 herbaceous peony cultivars identified 34 volatile compounds using headspace solid-phase microextraction (HS-SPME) coupled with GC-MS (Feng et al., 2016). In related research of tree peony (section Moutan of the genus Paeonia), Li et al. (2012) also analyzed volatile organic components in petals of 30 cultivars with HS-SPME/GC-MS. Using dynamic headspace analysis and ATD-GC/MS, Zhao et al. (2012b) found that floral fragrances of six tree peony 
cultivars had significantly different quality and quantity. Li (2013) adopted static headspace and GC-MS to analyze the content and type of volatile components in the flowers of four tree peony cultivars, providing a basis for the use of natural flavoring substances such as citronellol, geraniol, and linalool in the development and utilization of foods, drinks such as peony tea or peony wine, spices, and other natural additives.

Herbaceous peony flowers are a rich source of natural antioxidants and contain anthocyanins, flavonoids, and other active ingredients that have been shown to be healthy for humans, offering great potential for their use in the food, cosmetics, and pharmaceutical industry, as well as in other sectors (Hu et al., 2008; Jian et al., 2010; Zhou et al., 2011). Despite this, aromatic profiling of herbaceous peony is highly restricted, and only three cultivars have been preliminarily examined and analyzed by HS-SPME and GC-MS, namely, different flowering stages of 'Yang Fei Chu Yu' and 'Da Fu Gui' (Huang et al., 2010), and different floral organs (petals, petaloid stamens, pistils, and sepals) of 'Qiao Ling' and 'Yang Fei Chu Yu' (Song and Yu, 2017).

In this study, using DHS and ATD-GC/MS, we identified and quantitatively analyzed the aromatic compounds in the volatile component of 30 herbaceous peony cultivars, covering the 'Lactiflora' group (1-16 in Fig. 1), the 'Hybrid' group (17-28 in Fig. 1), and the 'Itoh' group (29 and 30 in Fig. 1). In addition, using the floral aromatic compounds as variables in hierarchical cluster analysis, we classified the fragrance patterns of herbaceous peony cultivars for the first time by combining these results with the identification of the main aromatic compounds and the results of sensory evaluation. This study provides a theoretical basis for the selection of new cultivars of aromatic herbaceous peony and the application of their flowers in the development of essences, spices, tea, and flavored food.

\section{Materials and Methods}

Plant materials. Thirty herbaceous peony cultivars were used in this study (Fig. 1). Plants were grown in Xiaotangshan Germplasm of Ornamentals and Breeding Base, National Engineering Research Center for Floriculture, Beijing, China.

Methods. At the end of Apr. 2016, we posted a study recruitment plan on the school bulletin board. In this sensory evaluation plan, the experimental procedure and purpose were displayed, and applicants

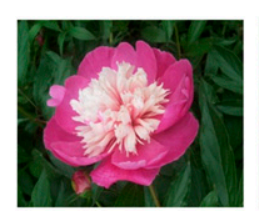

1 'Lian Tai'

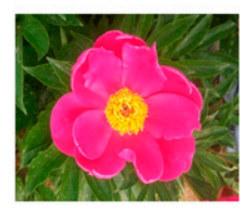

6 'Fen Yun Nu'

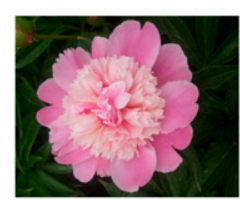

11 'Sorbet'

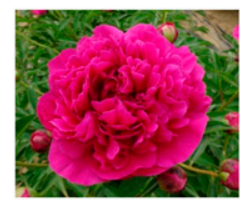

16 'Red Magic'

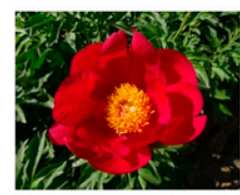

21 'Scarlet O'Hara'

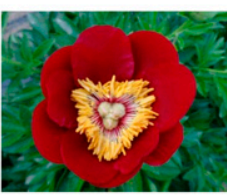

26 'Fairy Princess' 30: Itoh peony cultivar group. were clearly required not to be allergic to pollen. Verbal consent was obtained from all participants, 20 individuals 20-45 years of age who were evenly divided between men and women before the experiment. According to the flowering period of the tested cultivars, branches carrying flowers in full bloom were collected four times in batches while sampling the volatile components (between 10 and 25 May 2016). Branches were inserted in $20^{\circ} \mathrm{C}$ water for $1 \mathrm{~h}$ at room temperature. A sensory evaluation of the flowers of 30 herbaceous peony cultivars was carried out by the 20 participants. The members of this panel rated the fragrance of each sample based on a fourscale evaluation criteria: no fragrance $($ score $=0)$, light fragrance $(0<$ score $\leq 1)$, medium fragrance $(1<$ score $\leq 2)$, and intense fragrance $(2<$ score $\leq 3)$. For each panelist, the score of each cultivar was determined from the average of three scores, and the final score of each cultivar was determined by the average value of the average scores of all participants after outliers were eliminated by the Dixon's Q test (Efstathiou, 2006). Average scores and their respective sDs were calculated

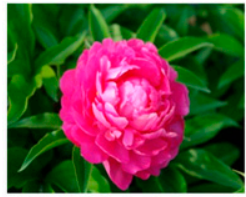

2 'Da Fu Gui'

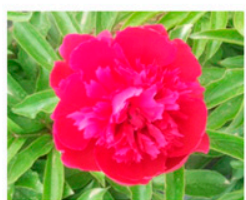

7 'Zhu Sha Pan'

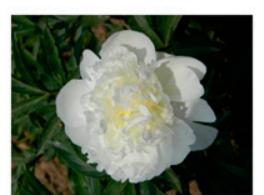

12 'Duchess de Nemours'

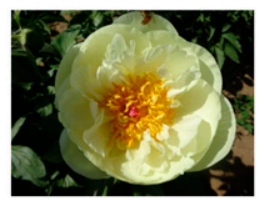

17 'Lemon Chiffon'

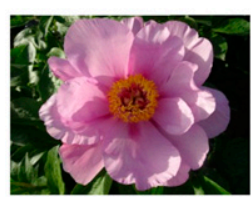

22 'May Lilac'

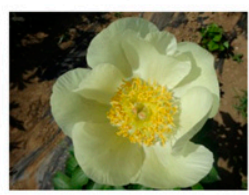

27 'Cream Delight'

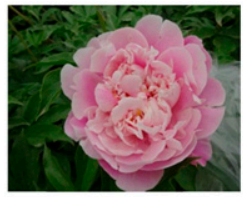

3 'Zhong Sheng Fen'

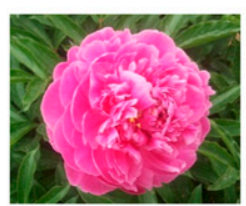

8 'Gao Gan Hong'

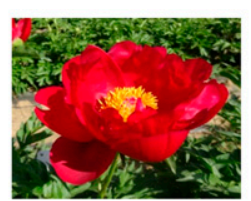

13 'Carina'

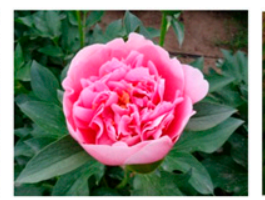

18 'Etched Salmon'

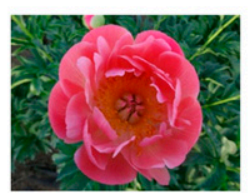

23 'Coral Sunset'

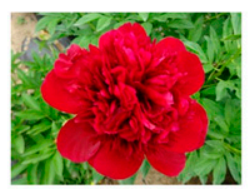

28 'Red Charm'

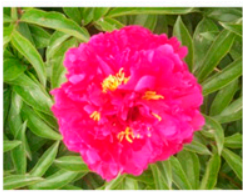

4 'Dong Fang Hong'

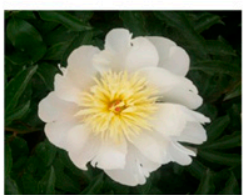

9 'Qiao Ling'

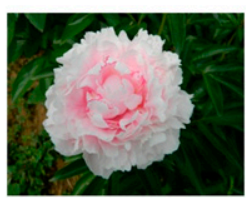

14 'Sarah Bernhardt'

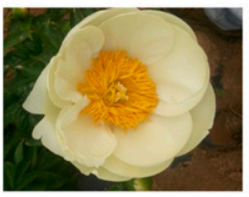

19 'Prairie Moon'

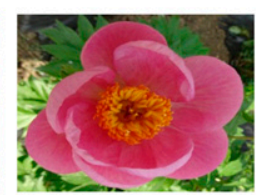

24 'Roselette'

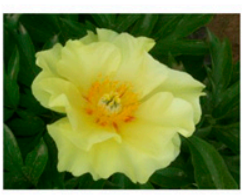

29 'Going Bananas'

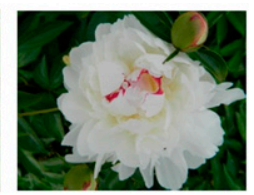

5 'Yang Fei Chu Yu'

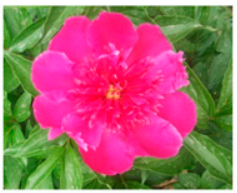

10 'Zi Feng Yu'

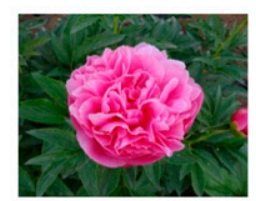

15 'Joker'

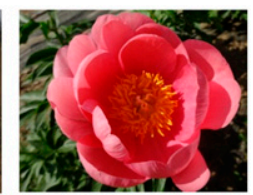

20 'Lovely Rose'

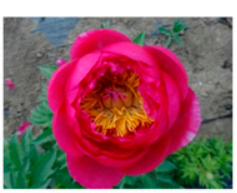

25 'Cytherea'

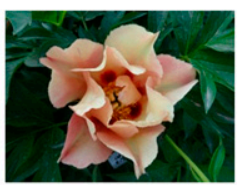

30 'Old Rose Dandy'

Fig. 1. Thirty herbaceous peony cultivars which were used for sensory evaluation and identifying and analyzing the aromatic compounds. 1 to 16: Lactiflora peony cultivar group; 17 to 28: Hybrid peony cultivar group; 29 and 
with IBM SPSS (version 21.0; IBM Corp., Armonk, NY). In addition, panelists compared the scents of the herbaceous peony cultivars with plant floral fragrance standards (Qingdao Jieshikang Biotechnology Corp., Qingdao, China), characterizing them as odorless, or with a lilac, rose, woody, or other scents.

Between 10 and 25 May 2016, plants with robust growth and at the full blooming stage were randomly selected. The volatile components of flowers in full bloom were collected by DHS. Sampling was performed in clear and windless weather, at a fixed time from 0800 to $1000 \mathrm{HR}$. Using a QC-4 type atmospheric sampler (Beijing Institute of Labor Protection Science, Beijing, China), the volatile components were extracted into a $355 \times 508-\mathrm{mm}$ aluminum foil gas sampling bag (Reynolds Metals Co., Richmond, VA) via a 200-mg stainless steel adsorption tube containing adsorbent (Tenax-TA; Beijing Kanglin Science \& Technology Co., Beijing, China). The sample volume flow rate was $0.3 \mathrm{~L} \cdot \mathrm{min}^{-1}$ and the gas production period was $20 \mathrm{~min}$. These and subsequent procedures were repeated three times for the fresh flowers of each cultivar from three independent plants. Samples were stored in a $5{ }^{\circ} \mathrm{C}$ ice box and quickly returned to the laboratory for testing.

The volatile components were subjected to ATD using a thermal desorber (Turbo Matrix 650; Perkin Elmer, Waltham, MA). Conditions were optimized in preliminary trials with helium (purity $\geq 99.999 \%$ ) as the carrier gas, $280 \mathrm{kPa}$ carrier pressure, $260{ }^{\circ} \mathrm{C}$ first-stage desorption temperature, $7 \mathrm{~min}$ desorption time, secondary desorption conditioned for $5 \mathrm{~min}$ at $180{ }^{\circ} \mathrm{C},-30{ }^{\circ} \mathrm{C}$ cold trap adsorption temperature, $45 \mathrm{~s}$ injection time, $250{ }^{\circ} \mathrm{C}$ transmission line temperature, and $230{ }^{\circ} \mathrm{C}$ four-way valve temperature. The analytical column used was DB-5MS, and the silica capillary column was $30 \times$ $0.25 \times 0.25 \mathrm{~mm}$. Chromatographic conditions were a split injection mode, 1:20 split ratio, $200{ }^{\circ} \mathrm{C}$ inlet temperature, and $40{ }^{\circ} \mathrm{C}$ (2 min hold) initial temperature rising at a rate of $4{ }^{\circ} \mathrm{C} \cdot \mathrm{min}^{-1}$ to $200{ }^{\circ} \mathrm{C}(2 \mathrm{~min}$ hold $)$ and finally raised to $240{ }^{\circ} \mathrm{C}$ at a rate of $5^{\circ} \mathrm{C} \cdot \mathrm{min}^{-1}(3 \mathrm{~min}$ hold $)$. The entire process took $55 \mathrm{~min}$ to complete. Mass spectrometry conditions included $1 \mathrm{kV}$ voltage, electron impact ionization mode, $200{ }^{\circ} \mathrm{C}$ ion source temperature, and $250{ }^{\circ} \mathrm{C}$ interface temperature.

The total ionic chromatogram of GC/MS was obtained by the following steps. Based on the National Institute of Standards and Technology standard spectral database and combined with relevant literature (Liang et al., 2010; Shu, 2005; Song et al., 2008; Zhou et al., 2008), compounds were identified by comparison using G1701DA ChemStation (Agilent Technologies, Santa Clara, CA). With a chirospecific chromatographic system, optically active substances such as D-limonene, $(E)$-3,5-dimethyl-1,6-octadiene, $(R)$-citronellol, $(Z)$-nerolidol, $(E)-(+)$-nerolidol, $(Z)$-3-hexenyl acetate, $(Z)$-3-hexen-1-ol, $(E)$ 2-hexen-1-ol, $(E)$-citral, $(E)$-rose oxide, and others were further identified. $\alpha$-Pinene ( $>99 \%$ purity; Fluka Chemical Corp., Ronkonkoma, NY) was selected as the external standard and ethyl acetate was used as the solvent (initial concentration was $\left.0.858 \mathrm{~g} \cdot \mathrm{mL}^{-1}\right)$. Four dilutions $(\times 100, \times 500, \times 1000$, and $\times 3000)$ of ethyl acetate were prepared with a concentration of 8.580 , $1.716,0.858$, and $0.286 \mu \mathrm{g} \cdot \mu \mathrm{L}^{-1}$, respectively. GC/MS analysis of the dilutions and samples was conducted under the same conditions to obtain a corresponding total ionic chromatogram. Using the different chromatographic peak areas and concentrations of the $\alpha$-pinene standard, a corresponding equation for the standard curve was obtained: $y=887,814.28 x-28,421.76$
( $x$ is the chromatographic peak area and $y$ is the concentration). The correlation coefficient $R^{2}$ was 0.9906 . Thereafter, the amount of floral fragrance released by each cultivar was determined, according to the ion chromatogram peak area of floral components in each sample.

Some cultivars were excluded from the hierarchical cluster of aromatic compounds because of the low amount of fragrance released and poor sensory evaluation results. Referring to Zhang and Dong (2004), the amount of aromatic compounds released was used as a variable in a hierarchical cluster analysis by using the cluster program in IBM SPSS, and Ward's method was used to obtain the cluster result. In each case, the Euclidean distance between clusters was chosen as a measurement of proximity. Simultaneously, using the release of floral components as the variable and peony cultivars as the case, IBM SPSS was used to analyze the scent similarity of 24 herbaceous peony cultivars by Pearson's correlation analysis, to examine the precision of the clustering algorithm. By considering the clustering results, major aromatic components, and the results of sensory evaluation, the 24 herbaceous peony cultivars were finally classified into defined fragrance patterns.

\section{Results and Discussion}

Four LeVels OF SENSORY EVAluAtion. As shown in Table 1, cultivars Carina, Joker, Red Magic, Etched Salmon, Lovely Rose, and Fairy Princess, which accounted for $20.0 \%$ of all cultivars, had no detectable fragrance, whereas 11 cultivars (Dong Fang Hong, Fen $\mathrm{Yu} \mathrm{Nu}$, Da Fu Gui, May Lilac, Roselette, Red Charm, Prairie Moon, Cytherea, Scarlet O'Hara, Coral Sunset, and Old Rose Dandy), which accounted for $36.7 \%$ of all cultivars, had a light fragrance with a nonobvious aroma. Cultivars Lian Tai, Zhong Sheng Fen, Gao Gan Hong, $\mathrm{Zi}$ Feng Yu, Sorbet, Sarah Bernhardt, and Lemon Chiffon, which accounted for $23.3 \%$ of all cultivars, had a medium fragrance level, whereas 'Yang Fei Chu Yu', 'Zhu Sha Pan', 'Qiao Ling', 'Duchess de Nemours', 'Cream Delight', and 'Going Bananas', which accounted for $20.0 \%$ of all cultivars, had an intense fragrance.

'Yang Fei Chu Yu' and 'Zhu Sha Pan' had a distinct orange blossom fragrance with a slight lemon fruit scent, 'Qiao Ling' and 'Duchess de Nemours' smelled like a typical rose, whereas 'Cream Delight' and 'Going Bananas' had a prominent lily fragrance with a slight fruity and woody scent. These 13 herbaceous peony cultivars had a distinguished aroma, making them useful as sources of fragrant flowers for ornamental or industrial purposes.

Aromatic Components. A total of 130 volatile organic components were identified according to the total GC/MS ionic chromatogram. Among them, 21 volatile components such as methanol, butane, 2,3-dimethylbutane, and others, despite having a pungent odor, are clearly defined as toxic and hazardous substances and were, therefore, excluded when identifying effective floral components. A very low content of 16 substances such as ethanol, fluorinated acetylene, dimethyl sulfide, and other common organic solvents were initially determined to be residues in the testing equipment and were, thus, excluded during the identification of floral components. It is presently not possible to determine whether 2,5-dimethyl-undecane, cis-7a-dimethyl-hexahydro-3a-2(3H)benzofuranone, 1,3,5-trimethoxybenzene, and 17 other volatile components are specific scent-related compounds. Therefore, 
Table 1. Aroma levels and description of floral scents in the flowers of 30 herbaceous peony cultivars.

\begin{tabular}{|c|c|c|c|c|}
\hline Cultivar no. ${ }^{z}$ & Score $($ mean \pm SD) & Description of scent & Aroma level & Total \\
\hline 13 & 0 & \multirow[t]{5}{*}{ Odorless } & \multirow[t]{5}{*}{ No fragrance $($ score $=0$ ) } & 6 \\
\hline 15 & 0 & & & \\
\hline 16 & 0 & & & \\
\hline 20 & 0 & & & \\
\hline 26 & 0 & & & \\
\hline 4 & $0.351 \pm 0.073$ & Fruity or grass scent & \multirow{7}{*}{$\begin{array}{l}\text { Light fragrance } \\
\qquad(0<\text { score } \leq 1)\end{array}$} & 11 \\
\hline 2 & $0.422 \pm 0.065$ & \multirow[t]{4}{*}{ Fruity } & & \\
\hline 22 & $0.474 \pm 0.081$ & & & \\
\hline 24 & $0.532 \pm 0.064$ & & & \\
\hline 28 & $0.611 \pm 0.075$ & & & \\
\hline 19 & $0.663 \pm 0.044$ & \multirow[t]{2}{*}{ Woody or lilac scent } & & \\
\hline 21 & $0.724 \pm 0.039$ & & & \\
\hline 8 & $1.302 \pm 0.069$ & \multirow{2}{*}{$\begin{array}{l}\text { Lily scent with slight woody scent } \\
\text { Orange blossom with a slight lemon } \\
\text { fruit scent }\end{array}$} & $(1<$ score $\leq 2)$ & \\
\hline 10 & $1.419 \pm 0.041$ & & & \\
\hline 3 & $1.604 \pm 0.085$ & \multirow[t]{3}{*}{ Rose scent } & & \\
\hline 11 & $1.677 \pm 0.052$ & & & \\
\hline 14 & $1.834 \pm 0.105$ & & & \\
\hline 17 & $1.923 \pm 0.035$ & Lily scent with woody scent or fruity scent & & \\
\hline 5 & $2.318 \pm 0.048$ & \multirow{4}{*}{$\begin{array}{l}\text { Orange blossom with a little lemon } \\
\text { fruit scent } \\
\text { Rose scent }\end{array}$} & \multirow{5}{*}{$\begin{array}{l}\text { Intense fragrance } \\
\quad(2<\text { score } \leq 3)\end{array}$} & 6 \\
\hline 7 & $2.442 \pm 0.077$ & & & \\
\hline 9 & $2.583 \pm 0.063$ & & & \\
\hline 12 & $2.601 \pm 0.055$ & & & \\
\hline 27 & $2.654 \pm 0.075$ & $\begin{array}{l}\text { Lily scent with slight fruity and } \\
\text { woody scent }\end{array}$ & & \\
\hline
\end{tabular}

${ }^{\mathrm{z}}$ Cultivar numbers assigned as described in Fig. 1.

72 compounds of 130 volatile organic components were identified as scent components in the 30 herbaceous peony cultivars (Table 2).

Relative to related reports on herbaceous peony by Feng et al. (2016), Huang et al. (2010), and Song and Yu (2017), our study identified nine new scent compounds: $\gamma$-terpinene, isocaryophyllene, $\alpha$-muurolene, $(E)-(+)$-nerolidol, isopentyl acetate, 3 methylbutyl 3-methylbutanoate, 3-hexen-1-ol, propanoate, methyl eugenol, and 4-allylanisole. One reason may be that the aforementioned studies used sampling in which flowers were cut and inevitably produced a certain physiological and biochemical stress response that is likely to have interfered with the aroma composition, the synthesis mechanism, and normal release, resulting in some loss of fragrance (Jia et al., 2008b). Naturally, this conclusion needs to be proved by further experiments. However, the use of DHS, which represents live dynamic sampling, can better reflect the actual release of plant volatile aromatic components than cut flowers. Table 2 shows that the main scent compounds were phenylethyl alcohol, $\beta$-caryophyllene, linalool, $(R)$-citronellol, and nerol. The aromatic compounds of the 30 cultivars were mainly composed of terpenes, alcohols, and esters, with a small amount of ketones, aldehydes, ethers, and other components, whereas the level of arenes ranged widely among the cultivars (Table 3). These differences in aromatic components result in different sensory floral scents.

The intensity of floral scent is defined as the sum amount of total volatiles (Wright et al., 2005). The total amount of floral aroma released from the 30 herbaceous peony cultivars in this study differed significantly (Table 2 ). Based on a standard curve, 17 cultivars released 50-150 $\mu \mathrm{g}$ of aromatic compounds (Table 2), but 'Going Bananas' $(285.01 \mu \mathrm{g})$, 'Cream Delight' (173.38 $\mu \mathrm{g})$, 'Zhu Sha Pan' (170.83 $\mu \mathrm{g})$, 'Qiao Ling' (164.25 $\mu \mathrm{g})$, 'Duchess de Nemours' $(146.97 \mu \mathrm{g})$, and 'Yang Fei Chu $\mathrm{Yu}^{\prime}(143.33 \mu \mathrm{g})$ released more fragrance than the other cultivars, corresponding to the results of sensory evaluation (Table 1). These typically aromatic herbaceous peony cultivars can be used not only as breeding parents for deriving fragrant progeny [lessons may be learned from Lilium (Johnson et al., 2016)] but also have good potential value as essences, spices, or tea, and use in flavored food development and other fields that use aromas. However, the amount of fragrance released by cultivars Red Magic $(7.86 \mu \mathrm{g})$, Joker $(12.48 \mu \mathrm{g})$, Fairy Princess $(15.15 \mu \mathrm{g})$, Lovely Rose $(27.25 \mu \mathrm{g})$, Carina $(29.54 \mu \mathrm{g})$, and Etched Salmon $(31.95 \mu \mathrm{g})$ was too low to determine a specific floral scent.

Fragrance Patterns. Cultivars Red Magic, Joker, Fairy Princess, Lovely Rose, Carina, and Etched Salmon were excluded from the hierarchical cluster of aromatic compounds and the analysis of fragrance patterns because of their low amount of fragrance release and poor sensory evaluation results. Using IBM SPSS, the hierarchical clustering of the remaining 24 cultivars is shown as a dendrogram (Fig. 2). The results of Pearson's correlation analysis using IBM SPSS indicate that the scent similarity of 'Prairie Moon' and 


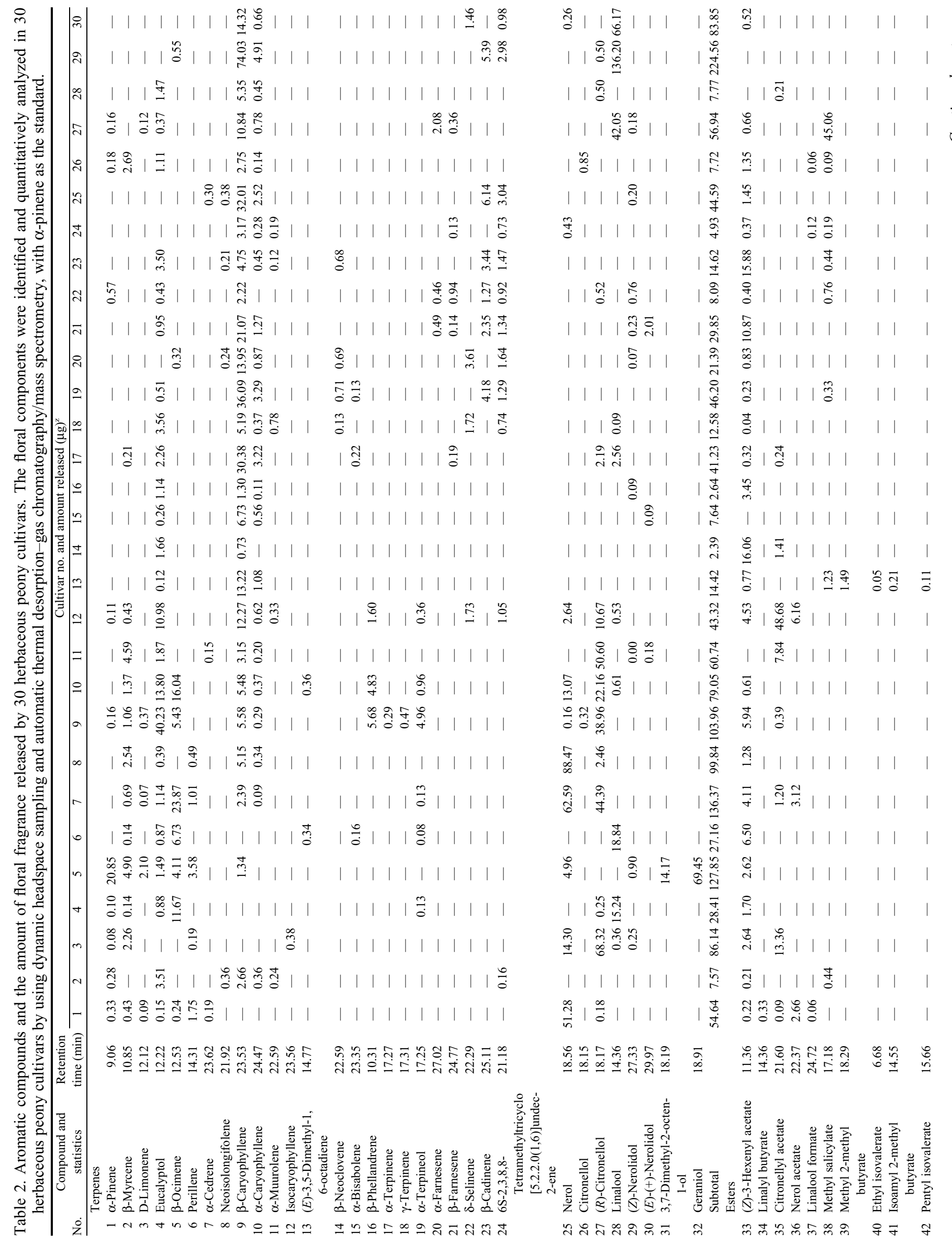




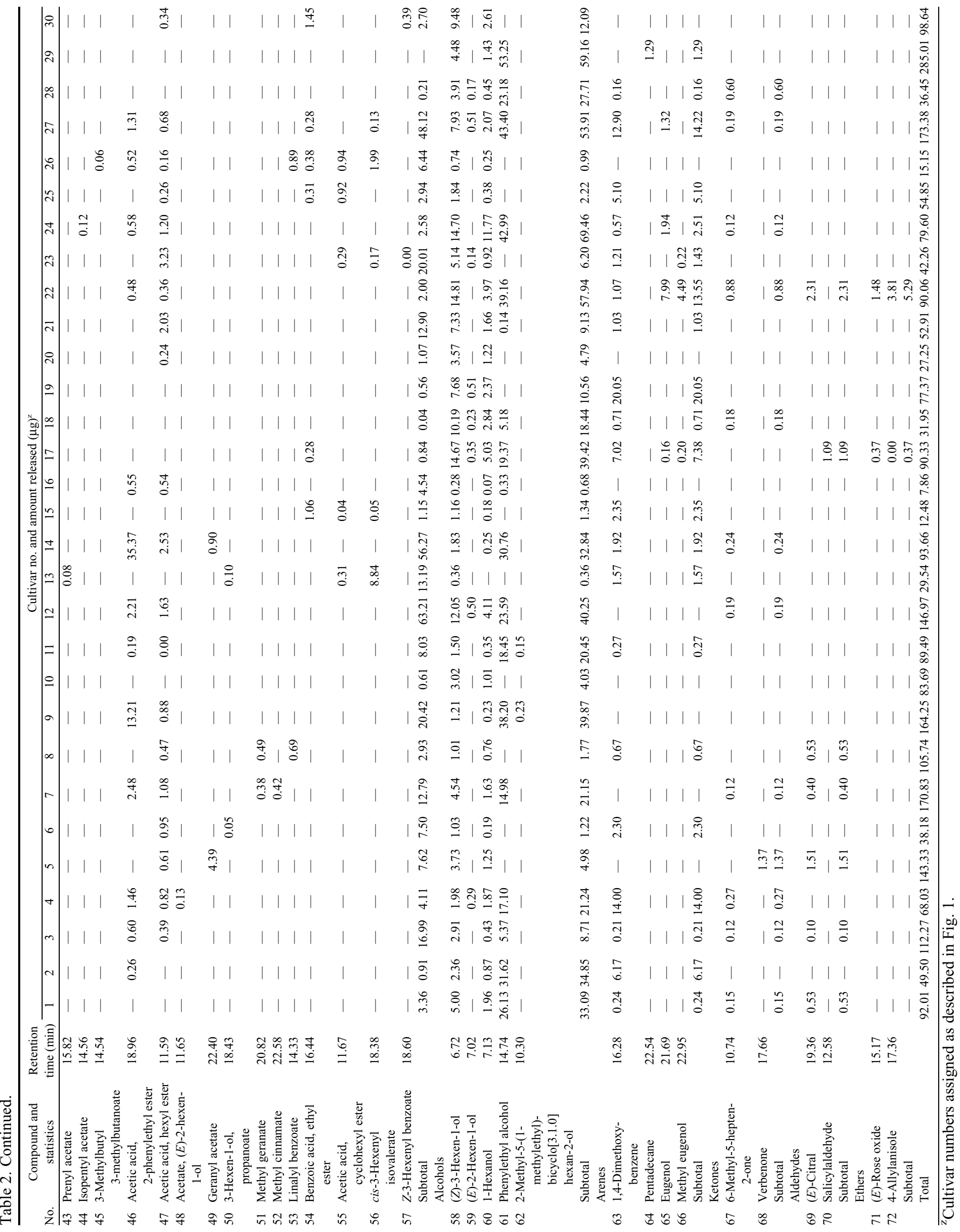


Table 3. The relative amount released of aromatic compounds emitted from 30 herbaceous peony cultivars. The floral components were identified and quantitatively analyzed in 30 herbaceous peony cultivars by using dynamic headspace sampling and automatic thermal desorption-gas chromatography/mass spectrometry, with $\alpha$-pinene as the standard.

\begin{tabular}{|c|c|c|c|c|c|c|c|c|}
\hline \multirow[b]{2}{*}{ Cultivar no. ${ }^{\mathrm{z}}$} & \multicolumn{8}{|c|}{ Relative amount released (\%) } \\
\hline & Terpenes & Esters & Alcohols & Arenes & Ketones & Aldehydes & Ethers & Subtotal \\
\hline 1 & 59.38 & 3.65 & 35.96 & 0.26 & 0.16 & 0.58 & 0.00 & 100.00 \\
\hline 2 & 15.29 & 1.84 & 70.40 & 12.46 & 0.00 & 0.00 & 0.00 & 100.00 \\
\hline 4 & 41.76 & 6.04 & 31.22 & 20.58 & 0.40 & 0.00 & 0.00 & 100.00 \\
\hline 5 & 89.20 & 5.32 & 3.47 & 0.00 & 0.96 & 1.05 & 0.00 & 100.00 \\
\hline 6 & 71.14 & 19.64 & 3.20 & 6.02 & 0.00 & 0.00 & 0.00 & 100.00 \\
\hline 8 & 94.42 & 2.77 & 1.67 & 0.63 & 0.00 & 0.50 & 0.00 & 100.00 \\
\hline 9 & 63.29 & 12.43 & 24.27 & 0.00 & 0.00 & 0.00 & 0.00 & 100.00 \\
\hline 10 & 94.46 & 0.73 & 4.82 & 0.00 & 1.06 & 0.00 & 0.00 & 100.00 \\
\hline 11 & 67.87 & 8.97 & 22.85 & 0.30 & 0.00 & 0.00 & 0.00 & 100.00 \\
\hline 12 & 29.48 & 43.01 & 27.39 & 0.00 & 0.13 & 0.00 & 0.00 & 100.00 \\
\hline 13 & 48.82 & 44.65 & 1.22 & 5.31 & 0.00 & 0.00 & 0.00 & 100.00 \\
\hline 18 & 39.37 & 0.13 & 57.72 & 2.22 & 0.56 & 0.00 & 0.00 & 100.00 \\
\hline 19 & 59.71 & 0.72 & 13.65 & 25.91 & 0.00 & 0.00 & 0.00 & 100.00 \\
\hline 20 & 78.50 & 3.93 & 17.58 & 0.00 & 0.00 & 0.00 & 0.00 & 100.00 \\
\hline 21 & 56.42 & 24.38 & 17.26 & 1.95 & 0.00 & 0.00 & 0.00 & 100.00 \\
\hline 22 & 8.98 & 2.22 & 64.33 & 15.05 & 0.98 & 2.56 & 5.87 & 100.00 \\
\hline 23 & 34.60 & 47.35 & 14.67 & 3.38 & 0.00 & 0.00 & 0.00 & 100.00 \\
\hline 24 & 6.19 & 3.24 & 87.26 & 3.15 & 0.15 & 0.00 & 0.00 & 100.00 \\
\hline 25 & 81.29 & 5.36 & 4.05 & 9.30 & 0.00 & 0.00 & 0.00 & 100.00 \\
\hline 26 & 50.96 & 42.51 & 6.53 & 0.00 & 0.00 & 0.00 & 0.00 & 100.00 \\
\hline 27 & 32.84 & 27.75 & 31.09 & 8.20 & 0.11 & 0.00 & 0.00 & 100.00 \\
\hline 28 & 21.32 & 0.58 & 76.02 & 0.44 & 1.65 & 0.00 & 0.00 & 100.00 \\
\hline
\end{tabular}

${ }^{\mathrm{z}}$ Cultivar numbers assigned as described in Fig. 1.

'Cytherea' [Pearson's correlation coefficient $(r)=0.88$ ], 'Roselette' and 'May Lilac' $(r=0.84)$, and 'Da Fu Gui' and 'Red Charm' $(r=0.78)$ was high (Table 4$)$, fully consistent with their clustering in Fig. 2. Therefore, the hierarchical clustering method used in this study is effective and reliable. By combining the results of cluster analysis with the identification of major aromatic components and the results of sensory evaluation, these 24 cultivars were divided into five aroma patterns: woody, fruity, lily, rose, and orange blossom scents.

Four herbaceous peony cultivars, with an average similarity of 0.65 , were grouped into the woody scent category: 'Prairie Moon', 'Cytherea', 'Scarlet O'Hara', and 'Coral Sunset'. The main aromatic component was $\beta$-caryophyllene, which has a woody aroma and is often used in the synthesis of acetyl caryophyllene and other more valuable spices (Liu, 2009). Therefore, 'Prairie Moon' $(36.09 \mu \mathrm{g})$, 'Cytherea' (32.01 $\mu \mathrm{g})$, and 'Scarlet O'Hara' $(21.07 \mu \mathrm{g})$, which contain a greater amount of $\beta$-caryophyllene than the other 21 cultivars, can serve in the aroma industry.

Cultivars Dong Fang Hong, Fen Yu Nu, Da Fu Gui, Red Charm, May Lilac, and Roselette had a fruity scent. As shown in Table 2, the main aromatic component of this group is phenylethyl alcohol (as high as $42.99 \mu \mathrm{g}$ in 'Roselette'), which has a pleasant fruit flavor and is widely used in the preparation of essences and spices (Sun et al., 2000). Furthermore, (Z)-3hexen-1-ol, $\beta$-caryophyllene, and eucalyptol were also detected. Eucalyptol is widely used in the chemical industry in the preparation of perfume; or as an oral freshener, skin cleanser, toothpaste, and air freshener; and other uses because of its fresh scent and potent bactericidal effect (Tian et al., 2007). The Pearson correlation coefficient between 'Dong Fang Hong' and 'Fen $\mathrm{Yu} \mathrm{Nu}$ ' $(r=0.75)$, 'Da Fu Gui' and 'Red Charm' $(r=0.78)$, 'May Lilac' and 'Roselette' $(r=0.84)$, and 'Red Charm' and 'Roselette' $(r=0.87)$ represents a fairly strong similarity and clustering relationship related to the fragrances of these six herbaceous peony cultivars (Table 4).

'Lemon Chiffon', 'Cream Delight', 'Old Rose Dandy', and 'Going Bananas' had a lily scent, with linalool as the major aromatic component (as high as $136.20 \mu \mathrm{g}$ in 'Going Bananas'). Linalool is the predominant aroma in Convallaria majalis and is used to produce essential oils and other flavors (Sun and Hao, 2009). In addition, high levels of $\beta$-caryophyllene were found in 'Going Bananas' $(74.03 \mu \mathrm{g})$ and 'Lemon Chiffon' (30.38 $\mu \mathrm{g})$, and phenylethyl alcohol in 'Going Bananas' (53.25 $\mu \mathrm{g})$ and 'Cream Delight' $(43.40 \mu \mathrm{g})$. Thus, the latter three cultivars have both a lily fragrance and a slightly woody and fruity scent. Curiously, the petals of four cultivars, Lemon Chiffon, Cream Delight, Old Rose Dandy, and Going Bananas, are all yellow or 


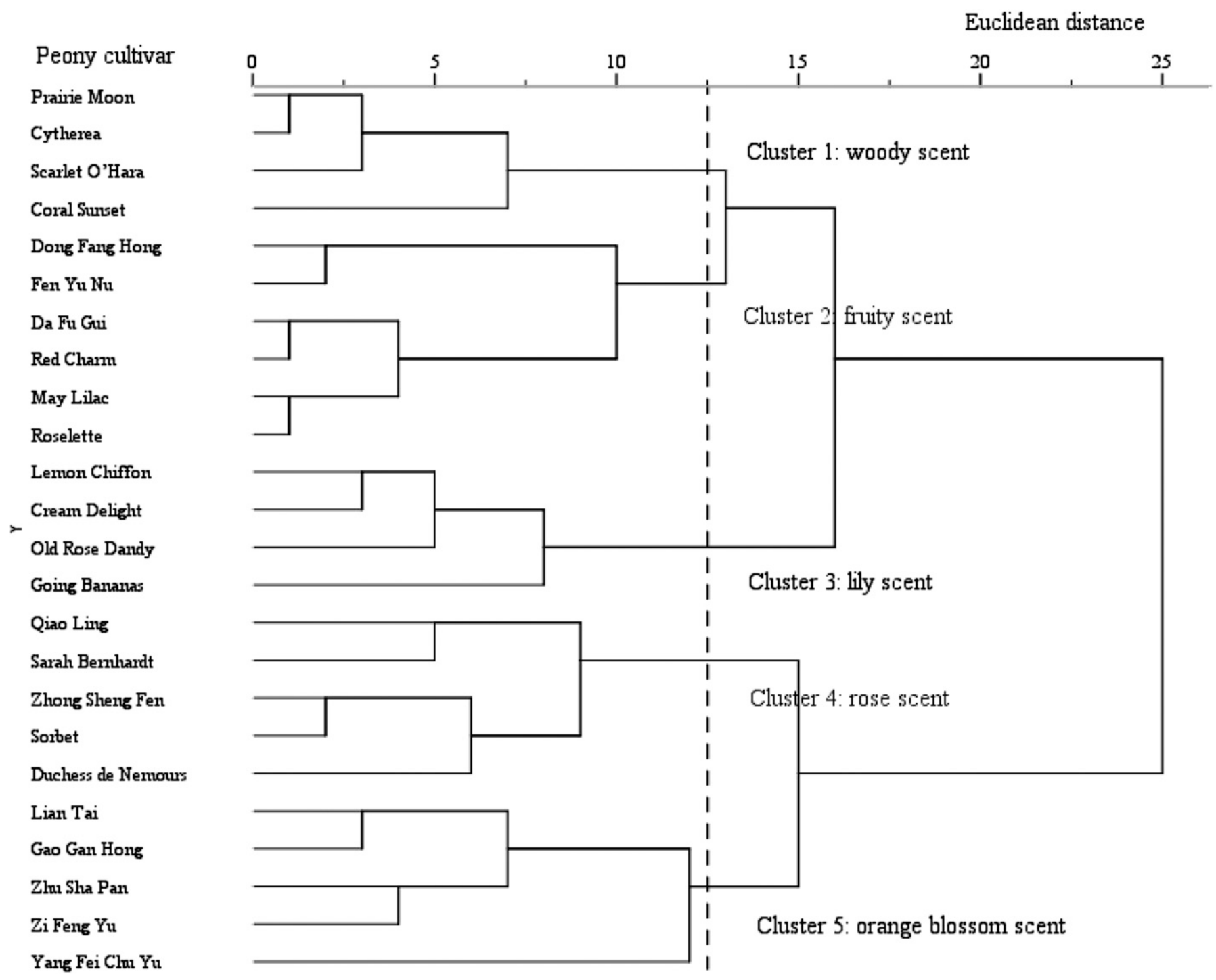

Fig. 2. Hierarchical cluster dendrogram of 24 herbaceous peony cultivars created by IBM SPSS (version 21.0; IBM Corp.). 'Red Magic', 'Joker', 'Fairy Princess', 'Lovely Rose', 'Carina', and 'Etched Salmon' were excluded from the hierarchical cluster of aromatic compounds because of the low amount of fragrance that they released and poor results of sensory evaluation. Ward's method was used to obtain the cluster result. In each case, the Euclidean distance between clusters was chosen as a proximity measurement. Simultaneously, using the release of floral components as the variable, and peony cultivars as the case, IBM SPSS was used to analyze the scent similarity rate of 24 herbaceous peony cultivars by Pearson's correlation analysis, to examine the precision of the clustering algorithm.

almost yellow. Even though the relationship between petal color and fragrance is uncertain, there is interest in breeding aromatic cultivars of herbaceous peony and distant hybrid breeding of yellow cultivars with this floral aromatic pattern.

Only cultivars Qiao Ling, Sarah Bernhardt, Zhong Sheng Fen, Sorbet, and Duchess de Nemours had a rose scent. In this odor, the main aromatic component of these five cultivars was $(R)$-citronellol [citronellyl acetate (as high as $68.32 \mu \mathrm{g}$ in Zhong Sheng Fen)], which emits a rose-like scent and is an essential ingredient in the production of perfumes, essences, spices, flavored foods, and rose tea (Cherri-Martin et al., 2007).

Cultivars Lian Tai, Gao Gan Hong, Zhu Sha Pan, Zi Feng $\mathrm{Yu}$, and Yang Fei Chu Yu had an orange blossom scent. Nerol, the main compound of this group, has a pleasant, fresh, and elegant aroma of orange blossom with some lemon-like fruity scent. It is the main component for the preparation of orange blossom, rose, and magnolia spices, and is widely used in the beverage and food industries, and deployed in high-grade essences ( $\mathrm{Li}, 2014)$. Nerol is also an intermediate and key raw material for the synthesis of other important spices, such as neroli essence, rose perfume, and magnolia oil ( $\mathrm{Li}$ et al., 2007a), and is presently in great demand worldwide (Ren and Zou, 2017). Moreover, the amount of aromatic components released by 'Zhu Sha Pan', an important parent material for the selection of fragrant herbaceous peony cultivars ( $\mathrm{Li}, 1999)$, is as high as $170.83 \mu \mathrm{g}$ and contains a high concentration of $\beta$-ocimene $(23.87 \mu \mathrm{g})$.

\section{Conclusions}

We identified the aromatic components and analyzed the fragrance patterns of 30 herbaceous peony cultivars, covering the 'Lactiflora', 'Hybrid', and 'Itoh' groups. This is the first time that such an analysis has been conducted for herbaceous 


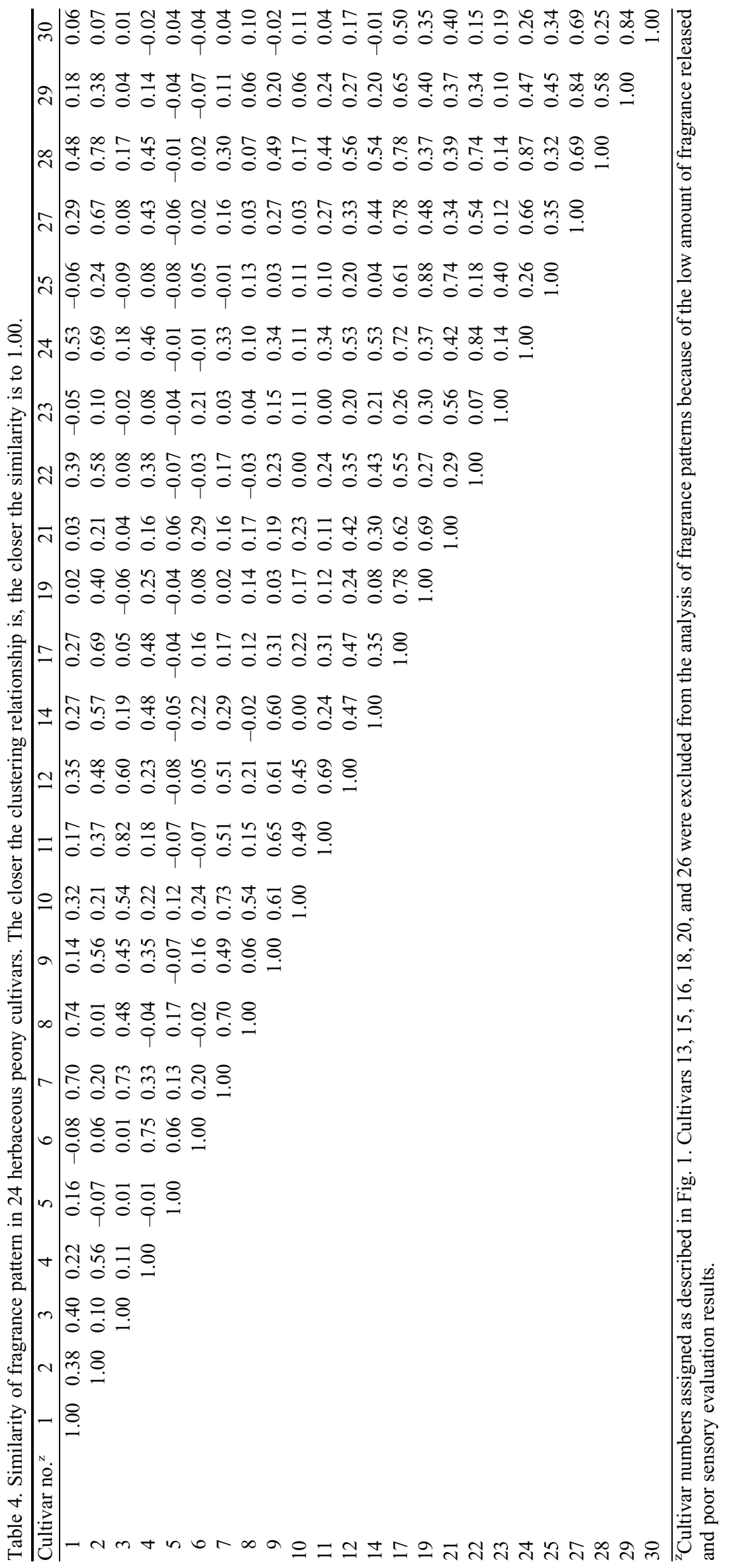


peony. Using DHS and ATD-GC/MS, 130 volatile organic components were detected. Among them, 72 compounds were identified as scent components. $\beta$-Caryophyllene, phenylethyl alcohol, linalool, $(R)$-citronellol (citronellyl acetate), and nerol were the major aromatic compounds. There was wide variation in the amount of aromatic compounds released by these 30 cultivars. 'Going Bananas', 'Cream Delight', 'Zhu Sha Pan', 'Qiao Ling', 'Duchess de Nemours', and 'Yang Fei Chu Yu' released a high amount of fragrance, allowing them to be used as breeding parents to derive fragrant progeny. By contrast, 'Red Magic', 'Joker', 'Fairy Princess', 'Lovely Rose', 'Carina', and 'Etched Salmon' released an undetectable amount of fragrance. Finally, cluster-based analysis, when combined with the major aromatic compounds and the results of sensory evaluation, allowed the remaining 24 herbaceous peony cultivars to be divided into and defined by five fragrance patterns: woody (major fragrance $\beta$-caryophyllene), fruity (phenylethyl alcohol), lily (linalool), rose [(R)-citronellol], or orange blossom scent (nerol). Additional research is needed to assess the aromatic biosynthetic pathway, expression patterns, and related physiological and biochemical mechanisms of different aromatic components of herbaceous peony.

\section{Literature Cited}

Cao, H., Z.G. Li, and D.L. Shen. 2009. GC/MS fingerprint analysis of Osmanthus fragrans in different varieties. Acta Hort. Sinica 36:391398.

Cheng, Y., C.H. Kim, D.I. Shin, S.M. Kim, H.M. Koo, and Y.J. Park. 2011. Development of simple sequence repeat (SSR) markers to study diversity in the herbaceous peony. J. Med. Plants Res. 5:67446751.

Cherri-Martin, M., F. Jullien, P. Heizmann, and S. Baudino. 2007. Fragrance heritability in hybrid tea roses. Scientia Hort. 113:177181.

Efstathiou, C.E. 2006. Estimation of type I error probability from experimental Dixon's "Q" parameter on testing for outliers within small size data sets. Talanta 69:1068-1071.

Fan, Z.Q., J.Y. Li, M. Tian, X.L. Li, and S. Ni. 2006. Preliminary studies of aromatic constituents among three species (variation) of Camellia. Acta Hort. Sinica 33:592-596.

Feng, L.G., Y.M. Li, L.X. Sheng, T.L. Li, D.Q. Zhao, and J. Tao. 2016. Comparative analysis of headspace volatiles of different herbaceous peony cultivars. J. Essential Oil Bearing Plants 19:167-175.

Feng, L.G., L.X. Sheng, L.Y. Zhao, X.Y. Yu, D.W. Sao, and X.D. He. 2008. Changes of the aroma constituents and contents in the course of Rosa rugosa flower development. Scientia Agr. Sinica 41:43414351.

Guo, X.F. and L.Y. Wang. 2005. Study on numerical taxonomy of Chinese cultivated herbaceous peonies and its related wild species. Acta Hort. Sinica 32:473-476.

Guo, X.F. and L.Y. Wang. 2007. Genetic relationship of partial peony germplasm resources with RAPD markers. Acta Hort. Sinica 34:1321-1326.

Hu, W.Q., H. Lu, W. Liu, J.X. Yuan, and D.M. Zhang. 2011. Paternity identification and genetic structure analysis on the wild population in Paeonia lactiflora. Acta Hort. Sinica 38:503-512.

$\mathrm{Hu}$, X.L., F.J. Yin, Q.F. Cheng, C.T. Zhu, and Z.Y. Song. 2008. Analysis of active constituents in peony flowers at different blossoming stages. Food Sci. 29:511-514.

Huang, X., C. Wang, X.H. Wang, X.Z. Sun, and X.F. Guo. 2010. Preliminary study of aromatic components in herbaceous peonies of 'Yang Fei Chu Yu' and 'Da Fu Gui'. Acta Hort. Sinica 37:817-822. Ishizaka, H., H. Yamada, and K. Sasaki. 2002. Volatile compounds in the flower of Cyclamen persicum, C. purpurascens and their hybrids. Scientia Hort. 94:125-135.
Jia, N., Q.Y. Shu, L.S. Wang, H. Du, Y.J. Xu, and Z.A. Liu. 2008a. Analysis of petal anthocyanins to investigate coloration mechanism in herbaceous peony cultivars. Scientia Hort. 117:167-173.

Jia, P.Y., L. Zhou, W.W. Guo, L.Y. Wang, and L. Dong. 2008b. Postharvest behavior and endogenous ethylene pattern of Paeonia suffruticosa cut flowers. Acta Hort. 768:445-450.

Jian, Z.Y., J.B. Yu, and W.Q. Wang. 2010. RP-HPLC determination of main chemical components in different parts and different harvest periods of Paeonia lactiflora. Acta Pharmaceutica Sinica 45:489493.

Jiao, F., Q. Liu, G.F. Sun, X.D. Li, and J.Z. Zhang. 2016. Floral fragrances of Hemerocallis (daylily) evaluated by headspace solidphase microextraction with gas chromatography-mass spectrometry. J. Hort. Sci. Biotechnol. 91:573-581.

Johnson, T.S., M.L. Schwieterman, J.Y. Kim, K.H. Cho, D.G. Clark, and T.A. Colquhoun. 2016. Lilium floral fragrance: A biochemical and genetic resource for aroma and flavor. Phytochemistry 122:103112.

Kamenetsky, R., A. Barzilay, A. Erez, and A.H. Halevy. 2003. Temperature requirements for floral development of herbaceous peony cv. 'Sarah Bernhardt'. Scientia Hort. 97:309-320.

Li, J.J. 1999. Chinese peony and herbaceous peony. China For. Publishing House, Beijing, China.

Li, Y.Y. 2013. Static headspace-GC-MS determination of volatile components of 4 species of peony. Phys. Test. Chem. Anal., Part B 49:334-336.

Li, C. 2014. Design, synthesis and characterization of fatty acid esters in food flavours and nutritional supplements. Nanyang Technol. Univ., Singapore, PhD Diss.

Li, S.S., L.G. Chen, Y.J. Xu, L.J. Wang, and L.S. Wang. 2012. Identification of floral fragrances in tree peony cultivars by gas chromatography-mass spectrometry. Scientia Hort. 142:158165.

Li, Q.G., Y.X. Liu, G.L. Wang, and W. Yan. 2007a. Progress in catalysts for selective hydrogenation of citral to nerol and geraniol. Modern Chem. Ind. 27:32-36.

Li, X., Y.G. Zhang, G.S. Zheng, R.M. Li, and S.X. Guo. 2007b. Studies on the senescence process and membrane-lipid peroxidation of cut peony flowers during the vase-holding. Acta Hort. Sinica 34:14911496.

Liang, R.J., Z.H. Liang, and S.D. Shi. 2010. GC-MS analysis of fennel essential oil and its effect on microbiology growth in rats' intestine. Afr. J. Microbiol. Res. 4:1319-1323.

Liu, S.W. 2009. Technical manual of synthetic perfumery. China Light Ind. Press, Beijing, China.

Liu, Q., G.F. Sun, J.Z. Zhang, and X.D. Li. 2015. Study on floral scent of the genus Hosta. Scientia Agr. Sinica 48:4323-4334.

Nakamura, K., K. Matsubara, H. Watanabe, H. Kokubun, Y. Ueda, N. Oyamaokubo, M. Nakayama, and T. Ando. 2006. Identification of Petunia hybrida cultivars that diurnally emit floral fragrances. Scientia Hort. 108:61-65.

Ray, H.A., C.J. Stuhl, and J.L. Gillett-Kaufman. 2018. Floral fragrance analysis of Prosthechea cochleata (Orchidaceae), an endangered native, epiphytic orchid, in Florida. Plant Signal. Behav. 13: e1422461.

Ren, H.T. and B. Zou. 2017. Analysis of volatile components and antioxidant activity evaluation of geranium essential oil and water. China Surfactant Deterg. Cosmet. 8:463-467.

Shi, G.A., X.F. Guo, G.H. Zhang, and M.Z. Bao. 2008. Physiological changes during florescence and flower senescence of Chinese peony. Acta Bot. Boreali-Occident. Sinica 28:506-511.

Shu, H.F. 2005. Handbook of new synthetic edible spices. Chem. Ind. Press, Beijing, China.

Song, C.W. and X.N. Yu. 2017. Analysis of the aroma component of different flower organs of two peony varieties. J. Beijing For. Univ. 39:92-99.

Song, G.X., Y.X. Yu, L.X. Wang, and Z.F. Chen. 2008. Techniques and examples of aroma analysis. Chem. Ind. Press, Beijing, China. 
Sun, J.C. and H.L. Hao. 2009. The efficacy of different slow-releasing linalool formulation to Blattella germanica. Chinese J. Vector Biol. Control 20:42-44.

Sun, B.G., F.P. Zheng, and Y.P. Liu. 2000. Perfumes and essences. China Petrochem. Press, Beijing, China.

Tian, Y.H., X.M. Zhang, T.S. Huang, K.X. Zou, and J. Zhou. 2007. Research advances on the essential oils from leaves of eucalyptus. Food Fermentation Ind. 33:139-143.

Wang, R.H., Y.L. Liu, and J.R. Li. 2005. Studies on the blossom physiology in the different development stage of peony and Chinese peony flower. Acta Hort. Sinica 32:861-865.

Wang, R.H., H.J. Zhao, R.Q. Pang, and J.R. Li. 2006. Effect of low temperature storage on senescence physiology of the Chinese herbaceous peony flowers. J. Northwest Sci.-Technol. Univ. Agr. For. 34:55-59.

Wei, X.X., Y. Liu, and R.F. Gao. 2007. Cyanide-resistant respiration and its role of Paeonia lactiflora cut flowers during storage time. J. Beijing For. Univ. 29:206-210.

Wright, G.A., A. Lutmerding, N. Dudareva, and B.H. Smith. 2005. Intensity and the ratios of compounds in the scent of snapdragon flowers affect scent discrimination by honeybees (Apis mellifera). J. Comp. Physiol. A Neuroethol. Sens. Neural Behav. Physiol. 191:105-114.

Xiong, M., M.Q. Zhou, L. Xiang, H. Wu, and L.Q. Chen. 2012. Analysis of essential oil in Chimonanthus praecox by GC-MS. J. Huazhong Agr. Univ. 31:182-186.

Xu, J., Y.Y. Li, C.S. Zheng, C. Wang, and Y.Q. Yu. 2012. Studies of aroma compounds in chrysanthemum in different florescence and inflorescence parts and aroma releasing. Acta Bot. Boreali-Occident. Sinica 32:722-730.

Yu, H.X., M. Wang, G.H. Liang, X.D. He, Z.Y. Gong, and W.J. Sunday. 2006. Pereliminary identification of relationship among cultivars of Paeonia lactiflora by ISSR analysis. Plant Physiol. Commun. 42:271-274.

Zhang, W.T. and W. Dong. 2004. Advanced tutorial for statistical analysis using SPSS. High Educ. Press, Beijing, China.

Zhang, H.X., Z.H. Hu, P.S. Leng, W.H. Wang, F. Xu, and J. Zhao. 2013. Qualitative and quantitative analysis of floral volatile components from different varieties of Lilium spp. Scientia Agr. Sinica 46:790-799.

Zhao, J., Z.H. Hu, P.S. Leng, H.X. Zhang, and F.Y. Cheng. 2012 b. Fragrance composition in six tree peony cultivars. Korean J. Hort. Sci. Technol. 30:617-625.

Zhao, Y.Q., H.T. Pan, Q.X. Zhang, C.B. Pan, and M. Cai. 2010. Fragrant compounds from Armeniaca mume flowers. J. Beijing For. Univ. 32:201-206.

Zhao, D.Q., J. Tao, C.X. Han, and J.T. Ge. 2012a. Flower color diversity revealed by differential expression of flavonoid biosynthetic genes and flavonoid accumulation in herbaceous peony. Mol. Biol. Rpt. 39:11263-11275.

Zhou, H.M., J.C. Qi, M.J. Dong, P. Li, and J.Q. Ma. 2008. Analysis of the volatile components in peony flowers by SPME-GC-MS. Chem. Analysis Meterage 17:21-23.

Zhou, C.H., Y. Zhang, Y.L. Sheng, D.Q. Zhao, S.S. Lv, Y. Hu, and J. Tao. 2011. Herbaceous peony as an alternative source of oleanolic and ursolic acids. Intl. J. Mol. Sci. 12:655-667. 